and he has thus disclosed much that was not known concerning them.

It might be thought that we would now be possessed of a thorough knowledge of what tumours themselves are like through the diligent observations on human growths made during the last hundred years; but the very conception of what a tumour is has been proved far off the mark by a recent discovery. The long-accepted definition has been that a tumour is an autonomous new growth. But Prof. C. B. Huggin's demonstration that many prostatic cancers which have metastasized stand still or disappear after removal of the patients' testicles shows that even the cells of malignant growths, of cancers ordinarily fatal, may not be truly independent in their behaviour. The cells of these prostatic cancers have been spurred on by the male sex hormones elaborated within the testicles and responsible for the normal activities of the prostatic epithelium. When not thus urged they in some instances are unable to grow, and in others are incapable even of maintaining themselves.

The meaning of this discovery far transcends its practical application; for it means that thought and endeavour in cancer research have been led astray by the idea that tumour cells are anarchic. Medical scientists have long been aware that even the worst cancers nearly all make some attempt at the customary tasks of the body. But no one had shown that neoplastic cells making such attempts at function might also respond in line of duty to normal physiological influences such as the hormones exert. How far this principle finds expression in tumour behaviour has yet to be learnt, and how successfully it can be utilized in combating tumours. The course of events with prostatic cancer justified the expectation that many mammary cancers would regress when the influence of the female sex hormone was lessened by removal of the ovaries or by injections of testosterone. While the results with women have not been very promising thus far, the fact that cancers of the male breast yield to orchidectomy or to stilbœestrol provides encouragement, for it makes plain the existence of factors of critical importance which have still to be understood and pressed into service.

At the moment the role of the hormones in the production and behaviour of tumours occupies many of the best investigators, and scarcely a month goes by without some discovery. For example, certain transplantable testicular tumours in mice will grow only if the new hosts are given cestrogen. Lipschuitz, working in Chile and following up a discovery made by Nelson, that uterine myomas appear in guinea pigs repeatedly injected with œstrogen, has now demonstrated that testosterone will prevent them from developing. Whether this has a practical bearing remains to be seen.

It is a curious fact that, while hormones play an important part in tumour production and behaviour, little has been found as yet that the vitamins do. This is not for lack of tests with them.

The dependence of some tumours on hormonal influences justifies the inference that the change of a normal cell to a tumour cell is far from meaning that a growth will arise; and direct proof on this point has for some while been available. It has been found that if the calorie intake of mice is cut down so slightly that the animals are only 5 per cent under weight, appearing fit instead of fat, carcinogenic agents induce no tumours on their skin. This is not because these agents have failed to bring about neoplastic changes; for if the animals are full-fed later, tumours start forth. The 'spontaneous' pulmonary growths to which mice of some strains are liable can also be prevented by cutting down the food. It would be worth while to know whether cancer has fallen off in the starving populations of Europe. At the moment much work is under way to learn whether modifications of the diet which involve no reduction of calories will hinder tumour growth.

But how shall the presence of hidden, incipient cancers be perceived? The hope that they may elaborate specific antigenic substances, and thus provide serological tests, goes back to the first years of immunology, and recently an antigen has been come upon; Kidd has demonstrated the existence of a distinctive antigenic substance in the BrownPearce rabbit carcinoma. No path has been opened as yet, though, by this discovery, for the substance has proved to be peculiar to the Brown-Pearce tumour, and serologically quite different from an antigen encountered in another kind of epidermoid carcinoms of the rabbit. On the basis of these findings one might perhaps look forward to a multitude of diagnostic blood tests, each for some tumour of special sort, but not to a single test which would help in the generality of human instances-those in which the doctor wants to know whether there is any tumour at all in his patient, its nature coming afterwards. Even the possibility of special tests is still problematic, since the production of antibodies in rabbits with the tumours just mentioned, a production necessary to serological tests, is inconstant despite the circumstance that every one of the growths themselves contains the specific antigen.

\section{FREQUENCY-MODULATION IN BROADCASTING}

$\mathbf{F}$ REQUENCY-MODULATION, or the technique of varying the frequency of radio waves for sig. nalling purposes, has been known and used for many years ; but it was not until Armstrong in the United States in 1936 pointed out the importance of wideband frequency-modulation on ultra-short waves, combined with a means for noise suppression, that the practical possibilities of the system for broadcasting purposes were realized. A considerable amount of development work on frequency-modulation technique took place in the United States before the War, and a number of broadcast transmitting stations have been erected and are using the frequency band 40-50 Mc./s. (wave-lengths 6-7.5 m.). It was not possible during the War to obtain much firsthand information as to the practical results obtained in the United States; and so in view of the probable importance of the system in improving both the overall coverage and the quality of reception in broadcasting, the B.B.C. decided just before the end of the War to conduct field trials in Great Britain. A discussion of the relative merits of frequency. modulation for broadcasting purposes, and of the first results of these trials, is given in an article by H. L. Kirke in the B.B.C. Quarterly (vol. 1, No. 2, July 1946).

In frequency-modulation technique, the radio carrier-wave is varied in frequency by an amount depending upon the amplitude of the modulating oscillation and at the frequency of the modulation, which for sound broadcasting is within the normal 
audible range. At the greatest amplitude of modula. tion, the change in carrier frequency is termed the deviation, and the value of this as used for broadcasting in the United States and likely to be adopted in Britain is $\pm 75 \mathrm{kc} . / \mathrm{s}$. In contrast with this system is that of amplitude-modulation, which is used in normal broadcasting, and in which the amplitude of the carrier is varied at the modulation frequency from zero to twice its normal unmodulated value, the frequency of the carrier wave remaining unaltered during the modulation process. Any increase of modulation amplitude beyond the above maximum value would result in distortion both at the transmitter and at the receiver. In the case of frequency modulation, there is no precisely defined point at which distortion sets in, so that if the transmitter and receiver circuits are suitably designed, a greater range of modulation can be achieved with the frequency- than with the amplitude-modulation system.

In order to render a frequency-modulated signal audible, the receiver uses a device known as a 'discriminator', which in effect converts it into an amplitude-modulated signal, which may then be detected by any of the usual methods. Since no change in amplitude of the carrier wave is required to convey intelligence, the transmitter can be operated under constant load conditions of high efficiency; and at the receiver a limiting device can be used to make it insensitive to amplitude changes, and thus limit the effects of receiver noise and electrical interference. While it would not be worth while using frequency-modulation on medium or long waves, because the band-width would have to be restricted, this does not apply to ultra-short waves, less than 10 metres in length; and the wide-band modulation can here be used to advantage with an extended audio-frequency range and improved quality of reception.

These advantages of the frequency-modulation system for broadcasting on ultra-short waves are discussed in some detail in the article referred to above, and illustrated by tabulated results and graphs applicable to frequencies of $45 \mathrm{Mc} . / \mathrm{s} .(6 \cdot 7 \mathrm{~m}$.) and $90 \mathrm{Mc} . / \mathrm{s}$. $(3 \cdot 3 \mathrm{~m}$.$) . For example, the electrical$ interference from motor-car ignition systems, which is a serious factor in the reception of ultra-short wave broadcasting, is much less serious with frequency. than with amplitude-modulation. Incidentally, in the course of experimental observations on this point made alongside a main arterial road, it was found to be extremely rare to get any interference from military vehicles, which have suitable radio-frequency suppressors fitted. The universal fitting of suppressors to motor vehicles would therefore increase the service area of a broadcasting transmitter very considerably. While the interference tests which have so far been made indicate that, on a frequency of $90 \mathrm{Mc} / \mathrm{s}$., the field-strength required to give satisfactory reception in the presence of a given amount of interference is only about one-third that required on $45 \mathrm{Mc}$. $/ \mathrm{s}$., it is to be noted that the field-strength obtained on the higher frequency may be less, due to greater attenuation and screening effects. The effective range is thus about the same on the two frequencies, and in both cases the use of horizontal polarization appears to be preferable.

The propagation of ultra-short waves is not greatly affected by the nature of the ground over which the waves pass, as is the case for medium and long waves ; but the shorter waves are more affected by ground contour, hills casting shadows and thereby degrading the reception conditions in valleys. Considerable advantage is obtained by placing the transmitter on high ground, with as high an aerial as possible. The meteorological conditions in the atmosphere also affect the propagation of ultra-short waves, causing them to be refracted and so to reach greater distances than would otherwise be the case. The B.B.C. has carried out surveys of field-strength on both 45 and $90 \mathrm{Mc}$./s. from transmitters located at the Alexandra Palace Television Station and at its Research Department, near Oxford. Typical results described and illustrated in the paper show the effect of ground contour and the fading that is encountered when the receiving point is at or beyond direct optical range from the transmitter.

The latter portion of Mr. Kirke's article is concerned with the planning of an ultra-short wave broadcasting service, and tables are given showing the coverage area to be expected on each of the two frequencies considered, and for four grades of service between first-class reception in an urban area to second-class reception in quiet rural surroundings. Confirmation of these calculations is being obtained by the provision of practical data on the reception of frequency-modulation in the home, in the course of special tests in which members of the staff of the B.B.C. co-operate. It will thus be realized that active consideration is being given to the possibilities of ultra-short wave broadcasting with the view of relieving the present congestion in the medium and long-wave bands, and providing a satisfactory frequency-modulation service of high quality throughout Great Britain.

\section{SCIENTIFIC CENTENARIES IN 1947}

\author{
By Engineer-Captain EDGAR C. SMITH, \\ O.B.E., R.N.
}

A REVIEW of the centenaries which occur in 1947 may well begin with a footnote to Prof. W. M. Smart's address to the Royal Astronomical Society on "John Couch Adams and the Discovery of Neptune", published in Nature of November 9 1946. With the publication of the facts connected with the discovery, the controversy began to subside, and at the Oxford meeting of the British Association in June 1847, Adams and Le Verrier met. The latter had been invited to England by Airy; and J. D. Forbes recorded how he dined at Prof. Powell's with a small but excellent party including Airy, Le Verrier and Adams. Afterwards Forbes went to the Observatory to see Neptune. "Fancy the interest," he wrote, "of seeing the new planet for the first time in company with its two discoverers! Of course, there could be no going to bed without seeing it, and accordingly as it rose late, I did not get back to my college till very nearly two in the morning." A month later Le Verrier was at Cambridge, and so was Otto Struve, and Murchison wrote, "Whilst walking across the quadrangle of Trinity, Leverrier on one arm and Struve on the other, we saw the Duke of Wellington coming towards us in his red gown and I at once said to the two great astronomers, 'Now, gentlemen, here is the opportunity'. Struve was overjoyed, saying it was the thing of all others he wished; but Leverrier turned on his heel and left us saying, 'Pardonnez, c'est plus fort que moi ; jo suis Francais'." 